\title{
Role of pneumococcal vaccination in prevention of pneumococcal disease among adults in Singapore
}

\author{
This article was published in the following Dove Press journal: \\ International Journal of General Medicine \\ 31 March 2014 \\ Number of times this article has been viewed
}

\author{
Philip Eng' \\ Lean Huat Lim² \\ Chian Min Loo ${ }^{3}$ \\ James Alvin Low ${ }^{4}$ \\ Carol Tan ${ }^{5}$ \\ Eng Kiat Tan ${ }^{6}$ \\ Sin Yew Wong ${ }^{7}$ \\ Sajita Setia ${ }^{8}$ \\ 'Philip Eng Respiratory and Medical \\ Clinic, Mount Elizabeth Medical \\ Center, ${ }^{2} \mathrm{Dr}$ Lim Lean Huat and \\ Associates Pte Ltd, ${ }^{3}$ Department \\ of Respiratory and Critical Care \\ Medicine, Singapore General Hospital, \\ ${ }^{4}$ Department of Geriatric Medicine, \\ Khoo Teck Puat Hospital, ${ }^{5}$ Rophi \\ Clinic, Mount Elizabeth Novena \\ Specialist Centre, Singapore, ${ }^{6} \mathrm{Kevin}$ \\ Tan Clinic for Diabetes, Thyroid, and \\ Hormones, Mount Elizabeth Medical \\ Center, ${ }^{7}$ Infectious Disease Partners \\ Pte Ltd, Gleneagles Medical Center, \\ ${ }^{8}$ Medical Affairs Department, Pfizer \\ Pte Ltd, Singapore
}

Correspondence: Dr Sajita Setia Pfizer Pte Ltd, I Science Park Road, \#04-0I The Capricorn, Science Park II, Singapore 117528

Tel +6564038754

Fax +65 67224188

Email sajita.setia@pfizer.com

\begin{abstract}
The burden of disease associated with Streptococcus pneumoniae infection in adults can be considerable but is largely preventable through routine vaccination. Although substantial progress has been made with the recent licensure of the new vaccines for prevention of pneumonia in adults, vaccine uptake rates need to be improved significantly to tackle adult pneumococcal disease effectively. Increased education regarding pneumococcal disease and improved vaccine availability may contribute to a reduction in pneumococcal disease through increased vaccination rates. The increase in the elderly population in Singapore as well as globally makes intervention in reducing pneumococcal disease an important priority. Globally, all adult vaccines remain underused and family physicians give little priority to pneumococcal vaccination for adults in daily practice. Family physicians are specialists in preventive care and can be leaders in ensuring that adult patients get the full benefit of protection against vaccine-preventable diseases. They can play a key role in the immunization delivery of new and routine vaccines by educating the public on the risks and benefits associated with vaccines. Local recommendations by advisory groups on vaccination in adults will also help to tackle vaccine preventable diseases in adults. Keywords: pneumococcal vaccine, pneumonia, invasive pneumococcal disease, adults
\end{abstract}

\section{Pneumococcal disease (PD) in adults}

Streptococcus pneumoniae or pneumococcus is an important cause of morbidity and mortality in adults and children worldwide. ${ }^{1} S$. pneumoniae is a common colonizer of the upper respiratory tract and often spreads to the surrounding mucosal tissue. It causes a wide spectrum of disease and is the most common cause of communityacquired pneumonia (CAP). ${ }^{2}$ In most patients, it causes noninvasive disease such as otitis media, sinusitis, and pneumonia. However, in a percentage of patients, the infection spreads into the blood stream resulting in invasive PD (IPD) manifesting as bacteremia, bacteremic pneumonia, or meningitis. The incidence of IPD varies substantially and is affected by factors such as socioeconomic status, age, immune status, genetic background, and geographical location. ${ }^{2}$ Pneumococcal pneumonia typically presents with chills, fever, malaise, dyspnea, and a productive cough. Untreated patients can progress to acute respiratory failure, septic shock, multiorgan failure, and death within several days from onset. ${ }^{3}$ In adults, there are a number of risk factors commonly implicated in the development of pneumococcal pneumonia including age, chronic lung disease, chronic heart disease, smoking, alcohol consumption, and previous hospitalization for pneumonia. ${ }^{4}$ 
The capsular polysaccharide of S. pneumoniae determines the virulence and provides the antigenic target for natural and vaccine-mediated antibody production. There are more than 90 immunologically distinct serotypes of S. pneumoniae, based on the chemical composition of the capsular polysaccharide. ${ }^{5}$ Transmission of S. pneumoniae occurs through direct contact or via fomites and is facilitated by overcrowding. Colonization begins within a few months of birth and continues throughout adolescence. ${ }^{6}$ The probability of adult colonization is directly related to the presence of younger children in the household as adults not exposed to children generally have a lower prevalence of $S$. pneumoniae. ${ }^{7}$ There are a limited number of epidemiological studies in Singapore, particularly in relation to serotype prevalence. ${ }^{8-13}$ The available seroprevalence data is summarized in Table 1.

Cardiac complications account for one-third of the treatment failure cases in hospitalized pneumonia patients. ${ }^{14}$ Numerous cardiac complications independently associated with increased short-term mortality have been reported during the course of CAP (Table 2). ${ }^{15,16}$ Although the cause-effect relationship between pneumonia and heart failure might be bidirectional, cardiac arrest occurs in up to $3 \%$ of patients admitted to hospital with $\mathrm{CAP} \cdot{ }^{17}$ In view of the high incidence of complications in patients with pneumonia and the effect on mortality, the possibility of improving the outcomes of these patients by prevention is extremely important. ${ }^{16}$

\section{PD burden}

In Singapore, pneumonia is the third leading cause of death (after cancer and heart diseases) and accounted for $16 \%$ of total deaths in $2011 .^{18}$ The incidence of pneumonia in adults increases with age all over the world. ${ }^{19} \mathrm{~A}$ US study of 46,237 elderly patients reported 18.2 cases per 1,000 person-years among patients aged 65-69 years, rising to 52.3 cases per 1,000 person-years among patients aged $\geq 85$ years. ${ }^{20}$ Previous estimates of mortality from CAP range from 5.1\% for hospitalized and ambulatory patients to $36.5 \%$ for patients in intensive care units. ${ }^{21}$

S. pneumoniae is the leading cause of vaccine preventable disease. It is estimated that there are 2-4 million cases of pneumonia per year in the US, with S. pneumoniae accounting for up to two-thirds of bacterial pneumonia cases. ${ }^{22}$ The World Health Organization estimates that 1.6 million die every year from PD. ${ }^{23}$ Although the number of IPD cases is similar in the very young and the very old, mortality in elderly patients is disproportionately high. ${ }^{24}$ Singapore made IPD a legally notifiable disease under the Infectious Diseases Act in $2010 .{ }^{25}$ However, there have been only two retrospective studies assessing the incidence of PD in adults in Singapore. An analysis by Low et al in hospitalized patients in Singapore examined 4,275 patient records with PD from 1995-2004. ${ }^{26}$ The authors found that the mean annual hospitalization rate was 10.9 per 100,000 population but was considerably higher in the young ( $<4$ years; $39 / 100,000)$ and in the elderly (>75 years; $95 / 100,000)$. The overall mortality rate in this study was $3.2 \%$. The pneumococcal meningitis mortality rate was $23.3 \%$ and the pneumococcal pneumonia mortality rate was $2.9 \%{ }^{26}$ An analysis of 192 adult cases of IPD in Singapore reported an attributable mortality of $21.4 \%$ between 2000-2007.27

S. pneumoniae antibiotic resistance has risen dramatically over the last 10-15 years in Singapore. There was a marked increase in antimicrobial nonsusceptibility in children in Singapore in 2008 compared to 1997 (penicillin 27.4\% versus $69.5 \%$; erythromycin $33.4 \%$ versus $78 \%$; clindamycin $24.5 \%$ versus $45.8 \%$; tetracycline $48 \%$ versus $67.8 \%$ ), and multidrug resistance (defined as nonsusceptibility to three or more classes of antibiotics) increased from $33.3 \%$ to $74.6 \%{ }^{11}$ Penicillin and macrolide resistance to S. pneumoniae are a serious concern worldwide, especially in Asian countries. ${ }^{28}$ Penicillin-resistant pneumococcal pneumonia is more common in patients with increased risk for mortality, such as those with comorbidities. ${ }^{29}$ The probability of penicillin and co-trimoxazole $S$. pneumoniae resistance is increased in human immunodeficiency virus (HIV)-infected patients, associated with receipt of long-term co-trimoxazole prophylaxis. Six serotypes (6A, 6B, 9V, 14, 19F, 23F) account for more than $80 \%$ of penicillin-resistant or macrolide-resistant S. pneumoniae worldwide. ${ }^{30}$

\section{Vaccines available in Singapore for prevention of PD Polysaccharide vaccines}

The 23-valent pneumococcal polysaccharide vaccine (PPSV23) was approved by the Health Sciences Authority (Singapore regulatory body) for use in Singapore in 1988. There are two PPSV23 registered in Singapore: Pneumo 23 by Sanofi Pasteur (Lyon, France), and Pneumovax 23 by MSD (Lyon, France). PPSV23 contains $25 \mu \mathrm{g}$ of each of the 23 pneumococcal polysaccharide antigens (Table 3). These 23 serotypes accounted for $75.4 \%$ of IPD infections in Singapore in 2012. ${ }^{13}$ A number of case-control studies have been conducted to assess the effectiveness of PPSV23 in preventing IPD in older adults. Vaccination effectiveness has ranged from $43 \%-81 \% .{ }^{31}$ PPSV23 has been found to be effective in healthy individuals $<75$ years of age, but 

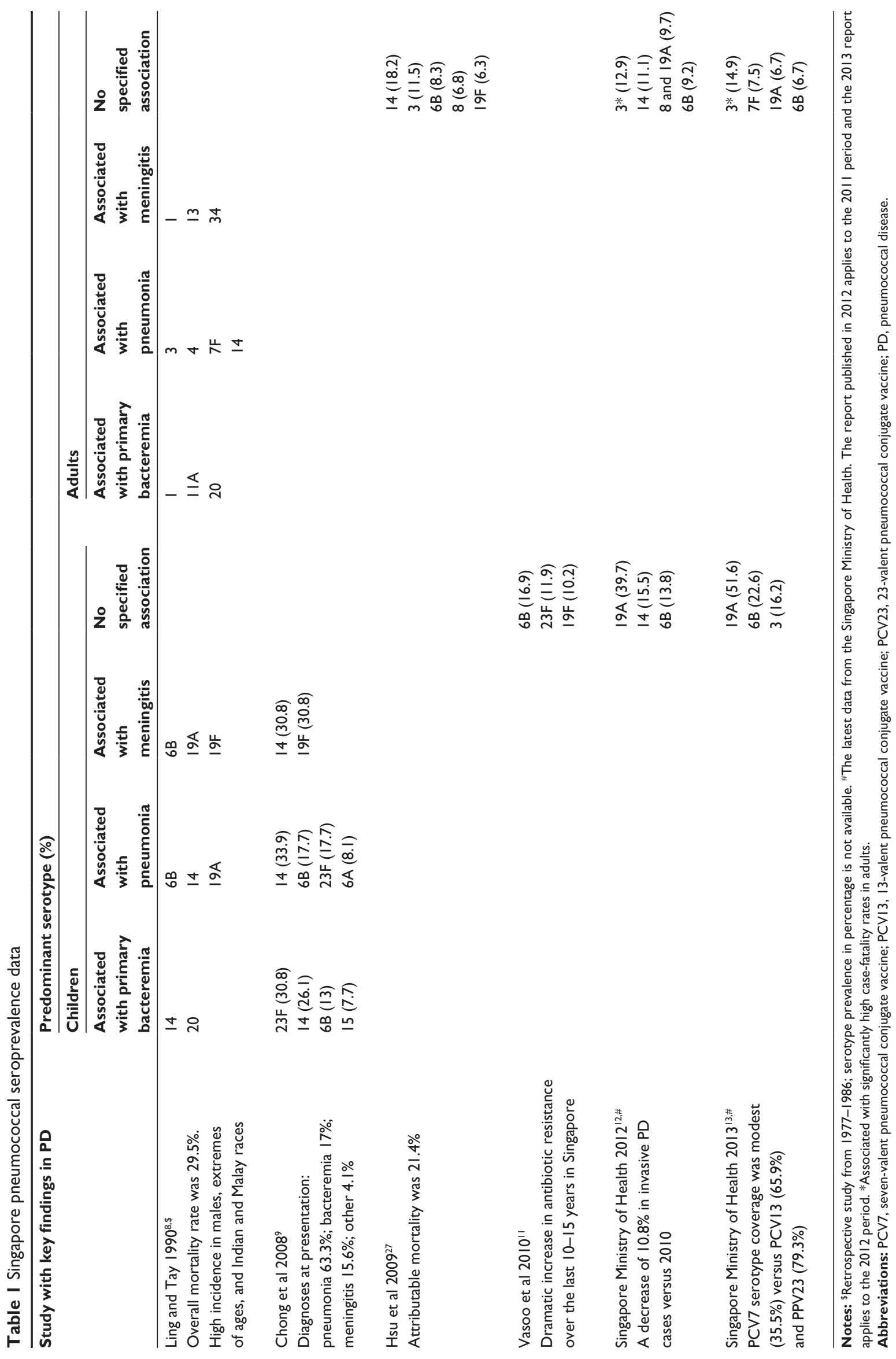
Table 2 Effects of pneumonia on the cardiovascular system

\begin{tabular}{|c|c|}
\hline $\begin{array}{l}\text { Vascular } \\
\text { endothelium } \\
\text { and peripheral } \\
\text { vessels }\end{array}$ & $\begin{array}{l}\text { Decreased peripheral vascular resistance in most } \\
\text { young adults, but increased peripheral vascular } \\
\text { resistance in up to one-third of middle-aged adults } \\
\text { (no data available for elderly patients); increased } \\
\text { concentrations of endothelin-I and adrenomedullin }\end{array}$ \\
\hline Myocardium & $\begin{array}{l}\text { Depression of left ventricular function; myocarditis; } \\
\text { increased concentrations of troponins, BNP, } \\
\text { and ANP }\end{array}$ \\
\hline Cardiac rhythm & Acute cardiac arrhythmias \\
\hline $\begin{array}{l}\text { Coronary } \\
\text { arteries }\end{array}$ & $\begin{array}{l}\text { Possible acute inflammatory changes in } \\
\text { atherosclerotic plaques; possible coronary } \\
\text { vasoconstriction }\end{array}$ \\
\hline $\begin{array}{l}\text { Pulmonary } \\
\text { circulation }\end{array}$ & Increased pulmonary artery pressures \\
\hline $\begin{array}{l}\text { Cardiac autonomic } \\
\text { function }\end{array}$ & Impairment of cardiovascular autonomic reflexes \\
\hline Coagulation & Increased procoagulant activity \\
\hline $\begin{array}{l}\text { Renal function } \\
\text { and fluid and } \\
\text { sodium balance }\end{array}$ & $\begin{array}{l}\text { Increased production of vasopressin; decreased } \\
\text { ACE activity; water retention; acute kidney injury }\end{array}$ \\
\hline
\end{tabular}

Note: Reprinted from The Lancet, Vol 38I, Corrales-Medina VF, Musher DM, Shachkina S, Chirinos JA, Acute pneumonia and the cardiovascular system, Pages 496-505, ${ }^{16}$ Copyright (C) 2013, with permission from Elsevier.

Abbreviations: $\mathrm{ACE}$, angiotensin-converting enzyme; ANP, atrial natriuretic peptide; BNP, B-type natriuretic peptide.

protection wanes after 5 years. ${ }^{31}$ Unlike conjugate vaccines, PPSVs have not been shown to interrupt carriage and therefore do not have the potential for herd effects. ${ }^{32-35}$ There is no discernible impact of PPSV23 on population incidence of IPD, and the older and high-risk individuals are less likely to benefit. ${ }^{36}$ However, based on vaccine efficacy estimates, PPSV23 is still considered a cost-effective intervention for the low-risk elderly. ${ }^{31,37}$

Overall, PPSV is quite a safe vaccine with minimal side effects, even in pregnancy. ${ }^{32}$ The Advisory Committee on Immunization Practices (ACIP) states that a pneumococcal vaccine may be administered at the same time as an influenza vaccine (by separate injection in the other arm) without an increase in side effects or decreased antibody response to either vaccine ${ }^{38}$ Recent studies indicated asthma as an independent risk factor for IPD, and the risk among persons with asthma was at least double that among controls. ${ }^{39}$ Similarly, smoking

Table 3 Serotypes included in pneumococcal vaccines

\begin{tabular}{ll}
\hline Vaccine & Serotypes included \\
\hline PPSV23 & I, 2, 3, 4, 5, 6B, 7F, 8, 9N, 9V, IOA, IIA, I2F, I4, I5B, \\
& I7F, I8C, I9A, I9F, 20, 22F, 23F, and 33F \\
PCV7 & $4,6 \mathrm{~B}, 9 \mathrm{~V}$, I4, I8C, I9F, and 23F \\
PCVI0 & $\mathrm{I}, 4,5,6 \mathrm{~B}, 7 \mathrm{~F}, 9 \mathrm{~V}, \mathrm{I4}, \mathrm{I8C}, 19 \mathrm{~F}$, and 23F \\
PCVI3 & $\mathrm{I}, 3,4,5,6 \mathrm{~A}, 6 \mathrm{~B}, 7 \mathrm{~F}, 9 \mathrm{~V}, \mathrm{I4}, 18 \mathrm{C}, 19 \mathrm{~A}, 19 \mathrm{~F}$, and 23F \\
\hline
\end{tabular}

Abbreviations: $P C V 7$, seven-valent pneumococcal conjugate vaccine; $P C V I 0$, tenvalent pneumococcal conjugate vaccine; PCVI3, I3-valent pneumococcal conjugate vaccine; PPSV23, 23-valent pneumococcal polysaccharide vaccine. was determined to be the strongest independent risk factor for IPD in immunocompetent, nonelderly adults. ${ }^{40}$ Consequently, in 2008, ACIP recommendations for PPSV23 were expanded for smokers and asthma patients. ${ }^{41}$ However, in some countries where PPSV23 is officially recommended, the estimated vaccination uptake is very low (around $2 \%$ in Switzerland) ${ }^{42}$ A recent survey in Switzerland among general practitioners revealed little priority for pneumococcal vaccination in daily practice, especially in comparison with the importance of other vaccinations, namely influenza. This low level of priority may be supported by the fact that general practitioners rarely ever experience a case of severe PD in their daily work. Insufficient evidence from PPSV23 clinical trials and existing epidemiologic data may have enhanced the lack of attention given to pneumococcal vaccination in general practice. ${ }^{42}$

\section{Conjugate vaccines}

In an effort to improve the immunogenicity of vaccines, pneumococcal conjugate vaccines (PCVs) were developed. The advantages of the conjugate vaccines are related to the way the antibodies are produced by the body. PCV induces a T-cell-dependent response which results in plasma cells producing immunoglobulin $\mathrm{G}$ but also produces memory B-cells. This T-cell-dependent response elicits immunological memory, and therefore primes the immune system for either natural exposure or subsequent booster vaccination. The latter observation may be considered a surrogate for exposure to the polysaccharide during a "natural" infection..$^{43}$ There is no decrease in the immune response seen with revaccination with conjugate vaccines, as they produce immunoglobulin $\mathrm{G}$ rather than just immunoglobulin M. ${ }^{44}$ Memory B-cells ensure boosting of the effect with revaccination. Memory B-cells are not produced in response to most free polysaccharide vaccines and, in fact, may be depleted post-vaccination, resulting in hyporesponsiveness. ${ }^{38,45}$

Seven-valent PCV (PCV7), which included the purified capsular polysaccharides of seven serotypes conjugated to a nontoxic variant of diphtheria toxin known as CRM197, was developed in 2000 (Table 3). ${ }^{46}$ PCV7 was licensed for children in Singapore in 2002 and PCV7 vaccination officially became part of the National Childhood Immunization Program (NCIP) in October 2009. ${ }^{13}$ After PCV7 was introduced in the US, rates of IPD caused by the seven serotypes have decreased substantially even among the unvaccinated population. ${ }^{47}$ The indirect benefits of vaccination, or herd effects, likely result from reduced nasopharyngeal carriage of pneumococcus in PCV7-vaccinated children and reduced transmission from children to unvaccinated children and 
adults. ${ }^{48}$ Surveillance data has shown reduction in IPD, pneumonia, and acute otitis media in young children after the introduction of PCV7 in many different geographic locations. ${ }^{23,30}$ A clinical trial in Malawi in predominantly HIV-infected adults documented significant protection from PCV7. In this study, survivors of pneumococcal pneumonia were randomized to receive PCV7 or placebo and were followed up thereafter for a mean of 1.2 years. The rates of protection were $74 \%$ in $\mathrm{HIV}$-infected persons. ${ }^{49} \mathrm{PCV} 7$ has also been shown to induce a superior immune response in patients with chronic obstructive pulmonary disease when compared with PPSV23. ${ }^{50}$

In order to provide improvements in serotype coverage and potentially reduce the remaining IPD burden, further improvements in conjugate vaccines were released. A tenvalent PCV using recombinant nontypeable Haemophilus influenza protein D as the conjugate was registered in Singapore and has been available since April 2010 (Table 3). A 13-valent PCV (PCV13) was approved for children in Singapore in 2010 and was introduced in the NCIP of Singapore in December 2011. ${ }^{13}$ PCV13 received Health Sciences Authority approval for expanded use in adults aged $>50$ years for the prevention of pneumonia and IPD on February 7, 2012.

PCV13 comprises "13" S. pneumoniae polysaccharide serotypes, including the existing seven in PCV7 and six additional serotypes (Table 3). The immunogenicity of PCV13 has been evaluated in a number of trials in healthy infants in comparator studies versus PCV7 and when coadministered with other vaccines. ${ }^{51}$ Noninferiority in terms of the proportion of responders 1 month after the final dose of the primary series of PCV13 versus PCV7 has been demonstrated for six of the seven common serotypes in one pivotal study ${ }^{52}$ and in five of the seven in another. ${ }^{53}$ The remaining six serotypes in PCV13 also demonstrated robust immune responses. ${ }^{52,53}$ Importantly, functional antibodies were elicited against all 13 serotypes contained in PCV13 after primary vaccination. ${ }^{52,53}$ Recently, the results of first randomized PCV13 versus PCV7 pediatric trial results were published. PCV13 resulted in lower acquisition and prevalence of nasopharyngeal colonization than PCV7 for four additional PCV13 serotypes, and serotypes 6C and 19F. It was comparable with PCV7 for all other common serotypes. ${ }^{10}$ Evidence for PCV13 protective effectiveness against IPD is also beginning to emerge in many industrialized countries such as England, Wales, Germany, and the US. ${ }^{53}$ These findings predict vaccine effectiveness through both direct and indirect protection. PCV13 immunogenicity and safety was also demonstrated either alone or with concomitant administration of a trivalent inactivated influenza vaccine in adults aged $\geq 65$ years who were naive to PPSV23. ${ }^{54}$

A meta-analysis of all available published data from controlled clinical trials of PCV (any valency) found the efficacy of PCV in the reduction of IPD was $89 \%$ for disease due to vaccine serotypes and $63 \%-74 \%$ for disease due to all serotypes. The efficacy to prevent acute otitis media by vaccine serotypes was $55 \%-57 \%$ and $29 \%$ to prevent otitis media involving all serotypes. However, the efficacy to prevent clinical pneumonia was only $6 \%-7 \%$, although it was $29 \%-32 \%$ to prevent radiograph-confirmed pneumonia. ${ }^{55}$

\section{PCVI3 for the prevention of PD in adults}

Despite multiple studies conducted for more than 30 years, the efficacy and effectiveness of PPSV23 in adults remain a subject of controversy. ${ }^{36}$ In a World Health Organizationcommissioned meta-analysis and a review of randomized controlled trials, the results of the randomized controlled trials of PPSV23 are consistent with a protective effect against IPD and all-cause pneumonia among generally healthy young adults and, to a lesser extent, in the general population of elderly people. ${ }^{56,57}$ However such trials have not demonstrated that PPSV23 is efficacious against either IPD or all-cause pneumonia in populations at higher risk, such as individuals with underlying conditions that increase their risk of PD or highly immunosuppressed individuals of any age. ${ }^{57,58}$

The availability of PCV13 in adults, however, sometimes creates a unique dilemma for clinicians as it is unusual to have availability of a new pediatric vaccine for a disease that is important in adults. ${ }^{59}$ Further complicating the situation is the lack of clear evidence regarding the relative benefits of PCV13 compared with PPSV23 in adults. However, several lines of evidence suggest the possibility that PCV13 may be more efficacious in adults than PPSV23. ${ }^{59}$ Relative absolute efficacy of the PCV13 and PPSV23 vaccines can be best demonstrated by randomized controlled trials; however, performing such trials is extremely difficult and expensive. Documenting an organism-specific effect for pneumococcal pneumonia can be quite challenging. Randomized trials of pneumococcal vaccine must be extremely large to have adequate statistical power. Cohort and case-control studies, although cheaper and logistically easier, can introduce bias from known and unknown confounders. ${ }^{59}$ Hence, the development of surrogate markers for vaccine efficacy has been critical. Enzyme-linked immunosorbent assay can be used to measure anti-pneumococcal immunoglobulin $\mathrm{G}$ 
antibodies, but cannot distinguish between functional and nonfunctional antibodies. ${ }^{60}$ Opsonophagocytic activity of pneumococcal polysaccharide antibodies in vitro is a measure of antibody functional activity and has been shown to correlate with immune protection more than enzyme-linked immunosorbent assays. ${ }^{61,62}$ This is because opsonophagocytic activity is the mechanism of protection against $\mathrm{PD}$, and hence it is the most appropriate assay for comparing antibody responses elicited by vaccines as well as for comparing the responses to various dosing regimens. ${ }^{43}$

The clinical program of PCV13 was designed to show that it could induce a T-cell-dependent response that could then be recalled or boosted by either natural exposure or a subsequent vaccination, or both. ${ }^{43}$ Pivotal Phase III clinical trials with PCV13 in adults have been performed in two populations aged $\geq 50$ years: those who were naive to previous vaccination (Study 004) 63,64 and those who had been previously vaccinated with PPSV23 but not within the past 5 years (Study 3005). ${ }^{65}$ An opsonophagocytic activity titer that correlates with protection has not been defined. Based on matching immunogenicity to efficacy results from several infant PCV7 or nine-valent PCV efficacy trials, a PCV13/PPSV23 geometric mean titer ratio was determined. ${ }^{63-65}$ If the lower $95 \%$ confidence interval of the ratio was $>0.5$ for serotypes in common, then the response was considered similar or noninferior. If, however, the lower $95 \%$ confidence interval was $>1.0$, then the response was considered to be statistically significantly higher or superior. ${ }^{43,63-65}$

Study $004^{63}$ was conducted in two age cohorts who had never received a pneumococcal vaccine previously: those aged 50-59 years and 60-64 years. At 1 month following the first dose, the response to a single dose of PCV13 was at least as good as the response to PPSV23 for all of the serotypes (noninferior) and statistically significantly higher for nine of the 13 serotypes. The overall superior response to a single dose of PCV13 was seen consistently throughout the clinical program. The response to a dose of PCV13 in those aged 50-59 years (mean age 54 years) was superior to the response in the 60-64 years cohort for eight of the twelve serotypes common to both vaccines and for serotype $6 \mathrm{~A}$, a serotype unique to PCV13, indicating the importance of age as a factor in the immune response. In the follow-up of Study $004^{64}$ conducted about 4 years later, the adults aged 60-64 years initially given PCV13 received PCV13 or PPSV23, and those initially given PPSV23 received another PPSV23. All adults aged 50-59 years were revaccinated with $\mathrm{PCV} 13$. Initial vaccination with
PCV13 established an immune state that resulted in recall of anti-pneumococcal responses upon subsequent vaccination with either a conjugate or free polysaccharide vaccine. In contrast, initial vaccination with PPSV23 resulted in an immune state in which subsequent PPSV23 administration yielded generally lower responses compared with the initial responses. Figure 1 shows the pattern of the immune response to serotype 1 , which is illustrative of the sequences for the majority of serotypes.

Study $3005^{65}$ enrolled PPSV23 exposed individuals (mean age 77 years) to receive either PCV13 or PPSV23 again. The pattern of the immune response was essentially the same as that seen in the PPSV23-naive adults (Study 004), although the magnitude of the response was somewhat lower in these older individuals. The response to PCV13 was at least as good as the response to PPSV23 and was statistically significantly higher for eleven of the 13 serotypes. Subjects were reimmunized 1 year later with PCV13, regardless of whether they had received PCV13 or PPSV23 the previous year. Subjects who had previously received a dose of PCV13 had a response that was at least noninferior to the first dose. In contrast, subjects who had received PPSV23 a year previous had a significantly reduced response to PCV13 (Figure 2 shows the pattern of

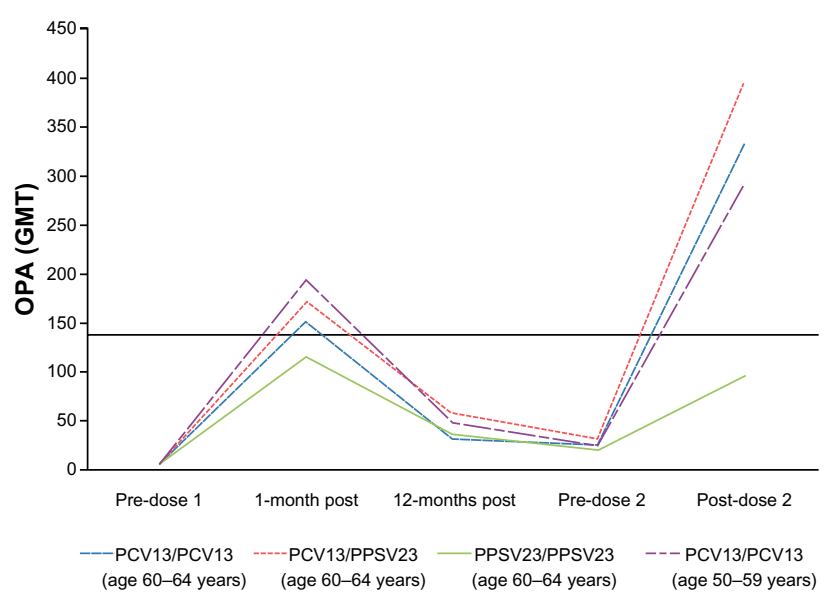

Figure I Functional immune responses for pneumococcal serotype I (GMT) in the pivotal noninferiority trial (Study 004) measured pre- and post-vaccination using a functional OPA assay.

Notes: Antibodies were determined before first vaccination (pre-dose I), I month after vaccination ( $\mathrm{I}$ month post), and 12 months after first vaccination ( 12 months post); and before the second vaccination 3-4 years later (pre-dose 2) and I month after the second vaccination (post-dose 2). An OPA titer that correlates with protection has not been defined. The dark straight line at GMT $\sim 40$ represents the minimum GMT peaks attained following first dose of PCVI3 to help understand amplification of response following revaccination. This demonstrates that PCVI3 primed the immune system for a booster response to subsequent vaccination with either vaccine. In contrast, the PPSV23 vaccine inhibited the response to a second dose of the same vaccine, and this was seen for all of the serotypes (serotype $\mathrm{I}$ is shown in figure as an example).

Reproduced from Paradiso PR. Pneumococcal conjugate vaccine for adults: a new paradigm. Clin Infect Dis. 2012;55(2):259-264, by permission of Oxford University Press. $^{43}$

Abbreviations: GMT, geometric mean titer; OPA, opsonophagocytic activity; PCVI3, I3-valent pneumococcal conjugate vaccine; PPSV23, 23-valent pneumococcal polysaccharide vaccine. 


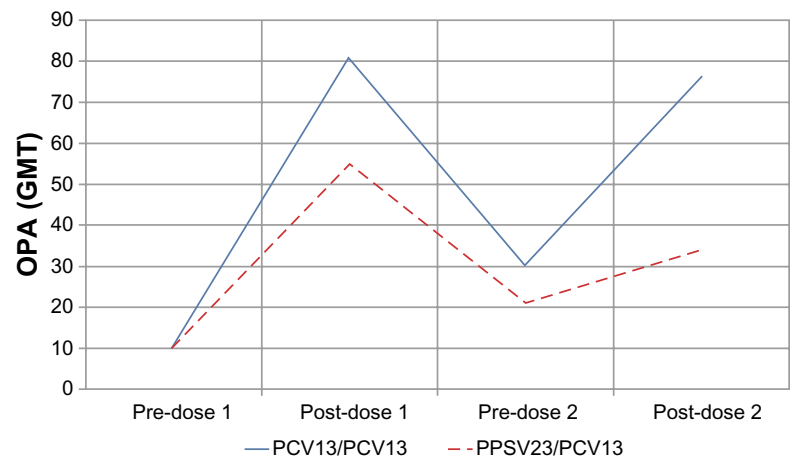

Figure 2 Functional immune responses for pneumococcal serotype I (GMT) in the pivotal noninferiority trial (Study 3005) measured pre- and post-vaccination using a functional OPA assay.

Notes: Study $3005^{65}$ enrolled PPSV23 exposed individuals (mean age 77 years) to receive either PCVI3 or PPSV23 again. Subjects were reimmunized I year later with PCVI3 regardless of whether they had received PCVI3 or PPSV23 the previous year. Antibodies were determined before first vaccination (pre-dose I), I month after vaccination (post-dose I), before the second vaccination (pre-dose 2), and I month after the second vaccination (post-dose 2). Study 3005 showed that PPSV23 also blunted the response to a dose of the conjugate vaccine (serotype $\mathrm{I}$ is shown in figure as an example).

Reproduced from Paradiso PR. Pneumococcal conjugate vaccine for adults: a new paradigm. Clin Infect Dis. 2012;55(2):259-264, by permission of Oxford University Press. ${ }^{43}$

Abbreviations: GMT, geometric mean titer; OPA, opsonophagocytic activity; PCVI3, I3-valent pneumococcal conjugate vaccine; PPSV23, 23-valent pneumococcal polysaccharide vaccine.

responsiveness to serotype 1 as an example). In summary, although Study 004 in vaccine-naive adults showed that PPSV23 blunted the response to a second dose of the same vaccine, Study 3005 showed that PPSV23 also blunted the response to a dose of the conjugate vaccine.

At the current time there is no clinical efficacy data regarding PCV13 efficacy in adults. The ongoing CAP Immunization Trial in Adults (CAPiTA) hopes to establish the efficacy of PCV13 in the prevention of pneumococcal CAP, non-bacteremic pneumococcal CAP, and IPD in community-dwelling elderly adults. ${ }^{66}$ Although CAPiTA is the largest vaccine efficacy placebo-controlled trial ever conducted in older adults worldwide, it does not include PPSV23 as an active comparator. The uncertainty surrounding the efficacy of PPSV for the prevention of noninvasive pneumococcal pneumonia made it very difficult to design an appropriate study and the required study size would likely have been prohibitive and made it logistically unfeasible. ${ }^{66}$ Recently, it was announced that CAPiTA has completed pneumonia case accrual and top-line results are expected in early $2014 .{ }^{67}$ The principal disadvantages of PCVs are their lesser breadth of coverage and the appearance of replacement strains. Although it is possible that with the widespread use of PCV13 new replacement strains will be those contained in PPSV23, it is also possible that altogether new strains may emerge not covered by any current vaccine. As per the Singapore Ministry of Health data published recently in 2013, the latest potential coverage rates of PCV13 and PPSV23 for adult IPD in 2012 were $67.9 \%$ and $75.4 \%$, respectively. ${ }^{13}$

\section{Is direct protection of adults with PCVI 3 required despite indirect benefits of herd effect?}

The strongest argument against the routine use of PCV13 in adults, whether they be healthy or immunocompromised, comes from the fact that routine $\mathrm{PCV}$ vaccination of children has also reduced the incidence of conjugate vaccine serotype disease in older adults. ${ }^{31}$ In PCV7-vaccinated children, the incidence of IPD was reduced by $>90 \%$. Not only was the effect in vaccinated children dramatic with widespread use of this vaccine, IPD in children who had not been vaccinated and in adults of all ages also decreased substantially. This phenomenon is called the herd effect: vaccinating a critical number of members of a population, especially with an agent that reduces colonization and infection, protects those who are unvaccinated. ${ }^{68}$ Herd effect is not the same as herd immunity. Herd immunity means that the immune system of unvaccinated persons has been affected by contact with a live vaccine strain or virus. For example, an unvaccinated person can develop immunity to poliovirus by being exposed to vaccine virus shed by a vaccinated person after receiving live oral polio vaccine ${ }^{69}$ Herd effect, unlike herd immunity, does not affect the immune system of unvaccinated persons. Unvaccinated individuals remain susceptible to infection if they come into contact with persons capable of transmitting disease ${ }^{69,70}$ Herd effect will not be seen immediately after the introduction of a new vaccine. For most infectious diseases, there is a threshold of vaccine coverage (uptake) that must be achieved for a herd effect to be seen. It takes time to reach that threshold. ${ }^{71}$ Herd effect is also dependent on the degree of vaccine coverage (uptake) in children. For example, herd effects with PCV have not been observed in countries with a low vaccine uptake. Furthermore, today's society is highly mobile. Even if an individual is a member of a population (herd) in which vaccine uptake is high, he or she will lose that protection upon leaving the herd and moving to another herd in which vaccine uptake is low. Furthermore, immunocompromised individuals are at much higher risk for IPD. ${ }^{72}$

Cohen et al studied the herd effects of pediatric use of PCV7 on the adult population with and without HIV infection. Declines in cases of IPD were observed in both groups; however, because of the high pre-PCV7 incidence of IPD in adults with HIV, the remaining disease burden was high despite a significant herd effect. ${ }^{73}$ From 2004-2007, the incidence of IPD 
in HIV-positive adults was 385 per 100,000. The incidence of IPD was approximately 40 times as high among adults with HIV infection as among adults without HIV infection, which called for more aggressive prevention measures. ${ }^{73}$ This study served as the basis for the ACIP recommendation on the use of PCV13 in adults who have immunocompromising conditions, functional or anatomic asplenia, cerebrospinal fluid leaks, or cochlear implants. ${ }^{74}$

A well-defined herd effect has also been observed in the US, UK, and Australia after introduction of PCV7 into their NCIPs. ${ }^{68,75,76}$ However, even after implementation of nationwide vaccination programs in France, Spain, and The Netherlands, analyses of epidemiological statistics in these countries showed significant serotype replacement and little evidence of a herd effect. ${ }^{77-80}$ It is hypothesized that serotype replacement has a disproportionate effect on herd effect due to the differing serotype distributions in the pediatric and adult populations. ${ }^{81}$ Therefore, the assumption that Singapore would experience a robust herd effect similar to that seen after implementation of PCV7 vaccination in the US $(30 \%$ of direct effect) was modified by the Singapore Ministry of Health. ${ }^{82}$ Given that negligible herd effects had been seen in most European investigations, there would be no guarantee of reductions in PD in the unvaccinated population; hence, there seems a need for direct protection of adults. The herd effect is also dependent on the degree of vaccine coverage (uptake) in children. For example, herd effects with PCV have not been observed in countries with a low vaccine uptake. ${ }^{71}$ Based on 2011 data from the Singapore National Immunization Registry, the vaccination uptake for pneumococcal vaccines (PCV7 and/or PCV13) was only $52 \%$ for children aged 1 year. ${ }^{13}$ So far, no significant changes in pneumococcal population dynamics related to herd effects of PCV13 have been observed in Singapore. ${ }^{13}$ However, PCV13 was included in the Singapore NCIP $<2$ years ago; hence, it might be also too early to document herd effects.

\section{International recommendations for the use of pneumococcal vaccines in adults}

In 2013, the US ACIP ${ }^{83}$ recommended vaccination with PPSV23 for all adults aged $\geq 65$ years and all adults $<65$ years with chronic lung disease, chronic cardiovascular diseases, diabetes mellitus, chronic renal failure, nephrotic syndrome, chronic liver disease (including cirrhosis), alcoholism, cochlear implants, cerebrospinal fluid leaks, immunocompromising conditions, functional or anatomic asplenia, residents of nursing homes or long-term care facilities, and adults who smoke cigarettes.
Persons with asymptomatic or symptomatic HIV infection should be vaccinated as soon as possible after their diagnosis. When cancer chemotherapy or other immunosuppressive therapy is being considered, the interval between PPSV23 and initiation of immunosuppressive therapy should be $\geq 2$ weeks. Vaccination during chemotherapy or radiation therapy should be avoided. Guidance on PPSV23 revaccination is outlined in Table 4.

There were no specific local/international recommendations for use of PCV13 in adults until June 2012 when the ACIP in the US made their recommendations for adults aged $>19$ years with immunocompromising conditions, functional or anatomical asplenia, cerebrospinal leaks, or cochlear implants (Table 5) ${ }^{74}$ In 2013, ACIP added guidance for the first time on the timing of administration of PCV13 relative to the PPSV23 in adults. ${ }^{83}$ In the conditions when

Table 4 Advisory Committee on Immunization Practices 2013 updates/highlights related to pneumococcal vaccination in adults

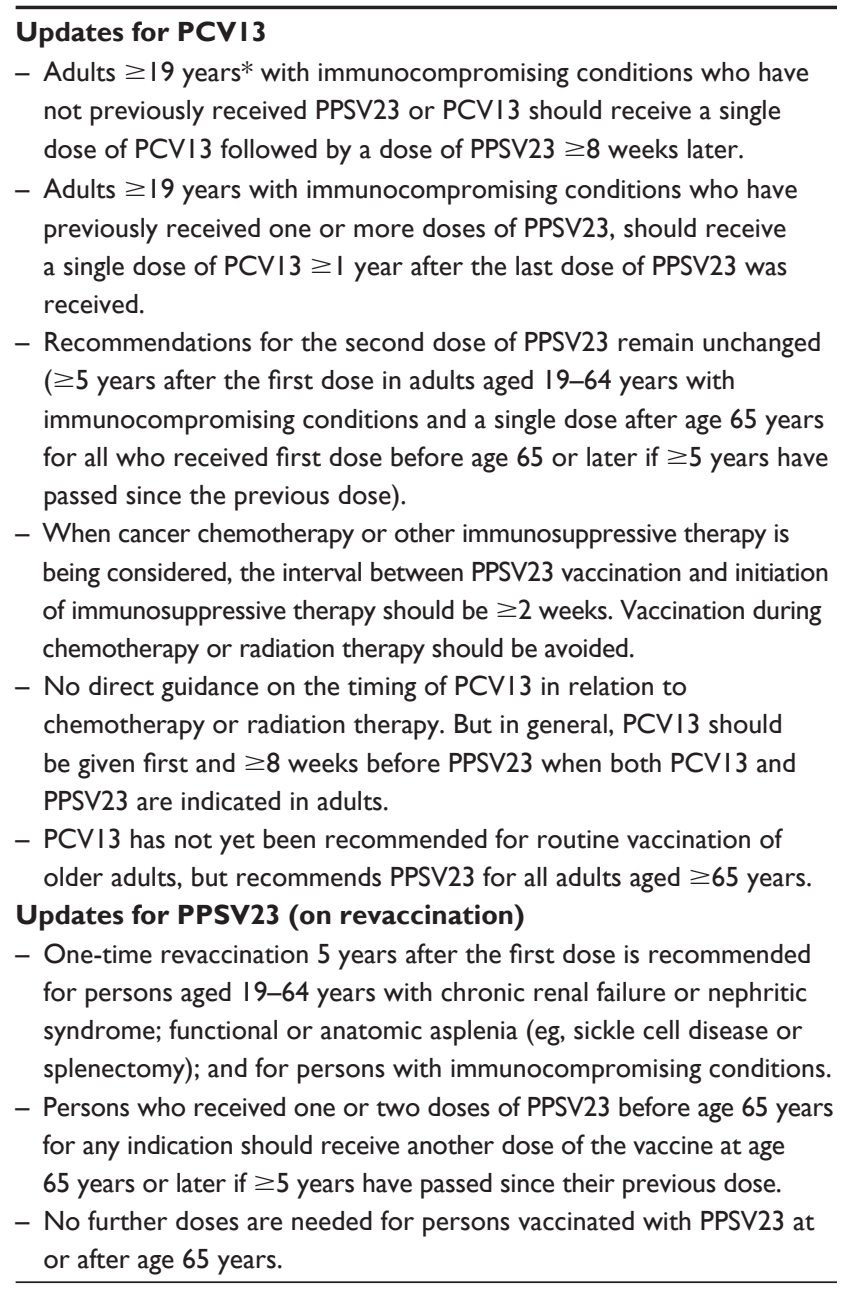

Notes: *PCVI3 is licensed by the US Food and Drug Administration and Singapore Health Sciences Authority for use in adults $\geq 50$ years of age only. Data from the Morbidity and Mortality Weekly Report, Centers for Disease Control and Prevention. ${ }^{83}$

Abbreviations: PCVI3, 13-valent pneumococcal conjugate vaccine; PPSV23, 23valent pneumococcal polysaccharide vaccine. 
Table 5 Advisory Committee on Immunization Practices 2012 recommendations on medical conditions or other indications for administration PCVI3 and PPSV23 for adults aged $\geq 19$ years, $*$ by risk group

\begin{tabular}{|c|c|c|c|c|}
\hline \multirow[t]{2}{*}{ Risk group } & \multirow[t]{2}{*}{ Underlying medical condition } & \multirow{2}{*}{$\frac{\text { PCVI3 }}{\text { Recommended }}$} & \multicolumn{2}{|l|}{ PPSV23 } \\
\hline & & & Recommended & $\begin{array}{l}\text { Revaccination } 5 \text { years } \\
\text { after first dose }\end{array}$ \\
\hline Immunocompetent & Chronic heart disease $^{\dagger}$ & & $\checkmark$ & \\
\hline \multirow[t]{7}{*}{ persons } & Chronic lung disease ${ }^{\S}$ & & $\checkmark$ & \\
\hline & Diabetes mellitus & & $\checkmark$ & \\
\hline & Cerebrospinal fluid leak & $\checkmark$ & $\checkmark$ & \\
\hline & Cochlear implant & $\checkmark$ & $\checkmark$ & \\
\hline & Alcoholism & & $\checkmark$ & \\
\hline & Chronic liver disease, cirrhosis & & $\checkmark$ & \\
\hline & Cigarette smoking & & $\checkmark$ & \\
\hline Persons with functional & Sickle cell disease/other hemoglobinopathy & $\checkmark$ & $\checkmark$ & $\checkmark$ \\
\hline or anatomic asplenia & Congenital or acquired asplenia & $\checkmark$ & $\checkmark$ & $\checkmark$ \\
\hline Immunocompromised & Congenital or acquired immunodeficiency" & $\checkmark$ & $\checkmark$ & $\checkmark$ \\
\hline \multirow[t]{10}{*}{ persons } & Human immunodeficiency virus infection & $\checkmark$ & $\checkmark$ & $\checkmark$ \\
\hline & Chronic renal failure & $\checkmark$ & $\checkmark$ & $\checkmark$ \\
\hline & Nephrotic syndrome & $\checkmark$ & $\checkmark$ & $\checkmark$ \\
\hline & Leukemia & $\checkmark$ & $\checkmark$ & $\checkmark$ \\
\hline & Lymphoma & $\checkmark$ & $\checkmark$ & $\checkmark$ \\
\hline & Hodgkin's disease & $\checkmark$ & $\checkmark$ & $\checkmark$ \\
\hline & Generalized malignancy & $\checkmark$ & $\checkmark$ & $\checkmark$ \\
\hline & latrogenic immunosuppression $* *$ & $\checkmark$ & $\checkmark$ & $\checkmark$ \\
\hline & Solid organ transplant & $\checkmark$ & $\checkmark$ & $\checkmark$ \\
\hline & Multiple myeloma & $\checkmark$ & $\checkmark$ & $\checkmark$ \\
\hline
\end{tabular}

Notes: *All adults aged $\geq 65$ years should receive a dose of PPSV23 regardless of previous history of vaccination with pneumococcal vaccine. ${ }^{\dagger}$ Including congestive heart failure and cardiomyopathy, excluding hypertension. IIncluding chronic obstructive pulmonary disease, emphysema, and asthma. "Including B- (humoral) or T-lymphocyte deficiency, complement deficiencies (particularly $\mathrm{Cl}, \mathrm{C} 2, \mathrm{C} 3$, and $\mathrm{C} 4$ deficiencies), and phagocytic disorders (excluding chronic granulomatous disease). **Diseases requiring treatment with immunosuppressive drugs, including long-term systemic corticosteroids and radiation therapy. Reprinted from Centers for Disease Control and Prevention (CDC). Use of I3-valent pneumococcal conjugate vaccine and 23-valent pneumococcal polysaccharide vaccine for adults with immunocompromising conditions: recommendations of the Advisory Committee on Immunization Practices (ACIP). MMWR Morb Mortal Wkly Rep. 2012;6I (40):816-819.74

Abbreviations: PCVI3, 13-valent pneumococcal conjugate vaccine; PPSV23, 23-valent pneumococcal polysaccharide vaccine.

both PCV13 and PPSV23 are indicated in adults, a dose of PCV13 should be given first. For those that require additional doses of PPSV23, the first such dose should be given no sooner than 8 weeks after PCV13 and $\geq 5$ years since the most recent dose of PPSV $23 .{ }^{83}$ Although ACIP recommends PCV13 for adults aged $\geq 19$ years with the specific medical conditions noted above. However, PCV13 is currently licensed by the US Food and Drug Administration and Singapore Health Sciences Authority for use among only in adults aged $\geq 50$ years (Table 4). ${ }^{83}$ On May 30, 2013, the Committee for Medicinal Products for Human Use, European Medicines Agency adopted a positive opinion recommending PCV13 for active immunization for the prevention of invasive disease caused by $S$. pneumoniae in adults $\geq 18$ years of age as well. ${ }^{84}$

\section{Cost-effectiveness of pneumococcal vaccines}

The costs associated with the treatment of CAP are considerable, although it is difficult to determine accurate cost-effectiveness data. A study conducted in a large urban hospital in the US showed that median hospital costs were $\$ 8,654$ and $\$ 9,441$ for CAP mediated by penicillinsusceptible and penicillin nonsusceptible $S$. pneumoniae, respectively. ${ }^{85}$ Several years ago, before the widespread introduction of PCV7 vaccination for children, the costeffectiveness of PPSV23 vaccination to prevent IPD was evaluated in ten Western European countries (PPSV23 was given concomitantly with influenza vaccine). PPSV23 was found to be acceptably cost-effective in all ten countries studied. However, while encouraging, these studies may not be relevant in countries that have introduced widespread vaccination of children with PCVs. ${ }^{31}$

There are few Asian cost-effectiveness studies of conjugate vaccines but all conclude that the inclusion of indirect or herd effects is essential to a positive assessment. PCV7 in children was found to be cost-effective when including herd effects in Hong Kong, ${ }^{86}$ but not cost-effective when herd effects were excluded in a Korean study ${ }^{87}$ Similarly, in the US, PCV13 has been estimated to be highly cost-effective if 
herd effects were included, ${ }^{88}$ but studies in The Netherlands excluding herd effects do not support this conclusion. ${ }^{81}$ A US cost-effectiveness study indicated PCV13 vaccination was favored compared with PPSV23 when substituted using the current recommendations, but the analysis was sensitive to assumptions about PCV13 effectiveness against non-bacteremic pneumococcal pneumonia and the magnitude of potential indirect effects from childhood PCV13 on pneumococcal serotype distribution. ${ }^{89}$ Another US study found that one dose of PCV13 is more cost-effective for immunocompromised individuals than previous vaccination recommendations and may be more economically reasonable than current recommendations depending on life expectancy and vaccine effectiveness in the immunocompromised. However, the findings were based on the assumption that both vaccines prevented IPD, but only PCV13 prevented non-bacteremic pneumonia. ${ }^{90}$

\section{Pneumococcal vaccine utilization for adults}

In Singapore, as well as globally, all adult vaccines remain underused. ${ }^{91-92}$ Rates of vaccination of adults have consistently fallen behind vaccination rates for children and adolescents. ${ }^{92}$ The Centers for Disease Control and Prevention estimated that 40,000-50,000 vaccine-preventable deaths occur every year in the US and estimates the health care burden of vaccine-preventable diseases at $\$ 10$ billion. ${ }^{92}$ The Centers for Disease Control and Prevention analyzed data from the 2010 National Health Interview Survey to assess adult vaccination rates and found them to be low for most routinely recommended vaccines. Only three vaccines are included in Healthy People 2020 - pneumococcal, herpes zoster, and hepatitis B (for health care personnel). These uptakes are well below the respective target levels. The uptake for pneumococcal vaccine in 2010 was only $59.7 \%$ for persons aged $>65$ years (Healthy People 2020 target: $90 \%$ ) and $18.5 \%$ for persons at high risk aged 18-64 years (Healthy People 2020 target: 60\%). ${ }^{92,93}$

In 2005, the World Health Organization estimated that 1.6 million people died of PD throughout the world, mainly the elderly and small children. ${ }^{23}$ In 2005 in the US, more than 40,000 cases and more than 4,400 deaths from IPD were estimated. More than half of these cases occurred in adults for whom the PPSV was indicated. ${ }^{94}$ Sustained declines in IPD have occurred among adults with acquired immunodeficiency syndrome in the US, but incidence remains high 7 years after PCV7 introduction. More aggressive efforts, including use of new PCVs in HIV-infected adults have been suggested to reduce IPD among HIV-infected adults. ${ }^{73}$ It is difficult to extrapolate from these conditions to conditions in the developed world, but the results suggest that, even in the developed world, patients with acquired immunodeficiency syndrome might respond to $\mathrm{PCV} .{ }^{36}$ Combination of the public seasonal flu vaccination campaign with a campaign for pneumococcal vaccination has been suggested to increase the pneumococcal vaccination rate. ${ }^{42}$

While there is less data in Asia regarding adult vaccine uptake compared to Western countries, several studies suggest there is significant PD in Singapore and vaccination should impact on reducing disease. ${ }^{9,26,27}$ The increase in the elderly population globally and in Singapore makes intervention in reducing $\mathrm{PD}$ an important priority. Family physicians are specialists in preventive care and can be leaders in ensuring that adult patients get the full benefit of protection against vaccine-preventable diseases. Standing order programs, which allow for vaccination without an individual physician order, are one of the most effective mechanisms to achieve high pneumococcal vaccination rates in adults. ${ }^{95-96}$ Since December 2010, one of the largest public hospitals piloted an innovative pre-discharge vaccination program for the first time in Singapore. This hospital-wide initiative integrates influenza and pneumococcal vaccination into standardized pre-discharge procedure for inpatients with appropriate medical indications. Patients and families may opt out, if desired. Pharmacists screen patients for indications and contraindications according to a pre-approved checklist and a vaccine is administered on the day of discharge by the nurse. This program enabled over 100 patients per month to get vaccinated who would not otherwise have received the vaccines. Such programs would be very useful if implemented nationwide to achieve maximum coverage and penetration for adult vaccination. $^{91}$

\section{Conclusion}

Improving the vaccination rates may contribute to a reduction in morbidity and mortality associated with PD affecting adults. Advances in vaccine production have provided both conjugate- and polysaccharide-based vaccines enabling wider serotype coverage and providing greater access to those in need. Studies indicate that conjugate vaccination of children can interrupt the transmission of pneumococci to adults and may prevent disease among adults, but there are also clear benefits of direct vaccination in adult populations. A significant burden of disease remains, particularly in immunocompromised and some immunocompetent adults with comorbidities; this remaining burden of disease may 
warrant a direct vaccination approach. It would be reasonable to conclude that for pneumococcal pneumonia prevention most high-risk adult patients may be best served by a combination of PCV13 and PPSV23, provided that PCV13 is given first. However, unless official country guidelines address potential advantages of PCV13 in adults especially in high-risk patients, physicians may be reluctant to consider it in routine clinical practice. There is a need for urgency about potential missed opportunities to prevent morbidity and death from PD in adults. Although reassessing local policy regarding pneumococcal vaccination in adults will require careful analysis of available information, advisory groups are encouraged to tackle this issue at the earliest opportunity.

\section{Acknowledgments}

The authors would like to acknowledge Bruce Mungall from UBM Medica Pte Ltd, Singapore for providing editorial and writing support, which was funded by Pfizer Pte Ltd, Singapore.

\section{Disclosure}

Dr Philip Eng, Dr Lean Huat Lim, Dr Chian Min Loo, Dr James Alvin Low, Dr Carol Tan, Dr Eng Kiat Tan, and Dr Sin Yew Wong received honoraria for participation in the advisory board on Singapore adult pneumococcal vaccination funded by Pfizer Pte Ltd, but received no compensation for their authorship of the article. Dr Sajita Setia is an employee of Pfizer Pte Ltd, Singapore.

\section{References}

1. Varon E, Mainardi JL, Gutmann L. Streptococcus pneumoniae: still a major pathogen. Clin Microbiol Infect. 2010;16(5):401.

2. Feldman C, Anderson R. Bacteraemic pneumococcal pneumonia: current therapeutic options. Drugs. 2011;71(2):131-153.

3. van der Poll T, Opal SM. Pathogenesis, treatment, and prevention of pneumococcal pneumonia. Lancet. 2009;374(9700):1543-1556.

4. Vila-Corcoles A, Ochoa-Gondar O, Rodriguez-Blanco T, Raga-Luria X, Gomez-Bertomeu F. Epidemiology of community-acquired pneumonia in older adults: a population-based study. Respir Med. 2009;103(2): 309-316.

5. Jacobs MR. Antimicrobial-resistant Streptococcus pneumoniae: trends and management. Expert Rev Anti Infect Ther. 2008;6(5):619-635.

6. Ekdahl K, Ahlinder I, Hansson HB, et al. Duration of nasopharyngeal carriage of penicillin-resistant Streptococcus pneumoniae: experiences from the South Swedish Pneumococcal Intervention Project. Clin Infect Dis. 1997;25(5):1113-1117.

7. Leino T, Auranen K, Jokinen J, Leinonen M, Tervonen P, Takala AK. Pneumococcal carriage in children during their first two years: important role of family exposure. Pediatr Infect Dis J. 2001;20(11): $1022-1027$.

8. Ling ML, Tay L. Epidemiology of pneumococcal infection in Singapore (1977-1986). Ann Acad Med Singapore. 1990;19(6):777-780.

9. Chong CY, Koh-Cheng T, Yee-Hui M, Nancy TW. Invasive pneumococcal disease in Singapore children. Vaccine. 2008;26(27-28): $3427-3431$.
10. Dagan $R$, Patterson $S$, Juergens $C$, et al. Comparative immunogenicity and efficacy of 13-valent and 7-valent pneumococcal conjugate vaccines in reducing nasopharyngeal colonization: a randomized double-blind trial. Clin Infect Dis. 2013;57(7):952-962.

11. Vasoo S, Singh K, Hsu LY, et al. Increasing antibiotic resistance in Streptococcus pneumoniae colonizing children attending day-care centres in Singapore. Respirology. 2011;16(8):1241-1248.

12. Ministry of Health, Singapore. Pneumococcal serotyping for surveillance of invasive pneumococcal diseases in Singapore, 2010-2011. Epidemiological News Bulletin. 2012;36(2):29-35.

13. Ministry of Health, Singapore. Laboratory data on surveillance of invasive pneumococcal diseases in Singapore, 2012. Epidemiological News Bulletin. 2013;39(2):34-39.

14. Aliberti S, Amir A, Peyrani P, et al. Incidence, etiology, timing, and risk factors for clinical failure in hospitalized patients with communityacquired pneumonia. Chest. 2008;134(5):955-962.

15. Corrales-Medina VF, Musher DM, Wells GA, Chirinos JA, Chen L, Fine MJ. Cardiac complications in patients with community-acquired pneumonia: incidence, timing, risk factors, and association with shortterm mortality. Circulation. 2012;125(6):773-781.

16. Corrales-Medina VF, Musher DM, Shachkina S, Chirinos JA. Acute pneumonia and the cardiovascular system. Lancet. 2013;381(9865):496-505.

17. Marrie TJ, Shariatzadeh MR. Community-acquired pneumonia requiring admission to an intensive care unit: a descriptive study. Medicine (Baltimore). 2007;86(2):103-111.

18. Principal causes of death [webpage on the Internet]. Singapore: Ministry of Health; 2012 [cited December 14, 2012]. Available from: http://www. moh.gov.sg/content/moh_web/home/statistics/Health_Facts_Singapore/ Principal_Causes_of_Death.html. Accessed September 3, 2013.

19. Fung HB, Monteagudo-Chu MO. Community-acquired pneumonia in the elderly. Am J Geriatr Pharmacother. 2010;8(1):47-62.

20. Jackson ML, Neuzil KM, Thompson WW, et al. The burden of community-acquired pneumonia in seniors: results of a populationbased study. Clin Infect Dis. 2004;39(11):1642-1650.

21. Fine MJ, Smith MA, Carson CA, et al. Prognosis and outcomes of patients with community-acquired pneumonia. A meta-analysis. JAMA. 1996;275(2):134-141.

22. Bartlett J. Treatment of community-acquired pneumonia. Chemotherapy. 2000;46(Suppl 1):24-31.

23. World Health Organization. Pneumococcal conjugate vaccine for childhood immunization - WHO position paper. Wkly Epidemiol Rec. 2007;82(12):93-104.

24. Centers for Disease Control and Prevention. Active Bacterial Core Surveillance Report. Emerging Infections Program Network: Group A Streptococcus, 2011. Atlanta, GA: Centers for Disease Control and Prevention; 2012. Available from: http://www.cdc.gov/abcs/reportsfindings/survreports/gas11.pdf. Accessed September 3, 2013.

25. List of infectious diseases legally notifiable under the Infectious Diseases Act [webpage on the Internet]. Singapore: Ministry of Health; 2010. Available from: http://www.moh.gov.sg/content/moh_web/ moh_corp_mobile/home/diseases_conditions/List_of_Infectious_ Diseases_Legally_Notifiable_under_the_Infectious_Diseases_Act. html. Accessed September 3, 2013.

26. Low S, Chan FL, Cutter J, Ma S, Goh KT, Chew SK. A national study of the epidemiology of pneumococcal disease among hospitalised patients in Singapore: 1995 to 2004. Singapore Med J. 2007;48(9):824-829.

27. Hsu LY, Lui SW, Lee JL, et al. Adult invasive pneumococcal disease pre- and peri-pneumococcal conjugate vaccine introduction in a tertiary hospital in Singapore. J Med Microbiol. 2009;58(Pt 1):101-104.

28. Song JH, Chang HH, Suh JY, et al. Macrolide resistance and genotypic characterization of Streptococcus pneumoniae in Asian countries: a study of the Asian Network for Surveillance of Resistant Pathogens (ANSORP). J Antimicrob Chemother. 2004;53(3):457-463.

29. Weinstein MP, Klugman KP, Jones RN. Rationale for revised penicillin susceptibility breakpoints versus Streptococcus pneumoniae: coping with antimicrobial susceptibility in an era of resistance. Clin Infect Dis. 2009;48(11):1596-1600. 
30. Lynch JP 3rd, Zhanel GG. Streptococcus pneumoniae: epidemiology and risk factors, evolution of antimicrobial resistance, and impact of vaccines. Curr Opin Pulm Med. 2010;16(3):217-225.

31. Fedson DS, Guppy MJ. Pneumococcal vaccination of older adults: conjugate or polysaccharide? Hum Vaccin Immunother. 2013;9(6): 1382-1384.

32. Fedson DS, Musher DM. Pneumococcal polysaccharide vaccine. In: Plotkin SA, Orenstein WA, Offit PA, editors. Vaccines. 4th ed. Philadelphia, PA: WB Saunders and Company; 2004:529-588.

33. Dagan R, Givon-Lavi N, Zamir O, et al. Reduction of nasopharyngeal carriage of Streptococcus pneumoniae after administration of a 9-valent pneumococcal conjugate vaccine to toddlers attending day care centers. J Infect Dis. 2002;185(7):927-936.

34. Fletcher MA, Laufer DS, McIntosh ED, Cimino C, Malinoski FJ. Controlling invasive pneumococcal disease: is vaccination of at-risk groups sufficient? Int J Clin Pract. 2006;60(4):450-456.

35. Cohen R, Levy C, Bingen E, Koskas M, Nave I, Varon E. Impact of 13-valent pneumococcal conjugate vaccine on pneumococcal nasopharyngeal carriage in children with acute otitis media. Pediatr Infect Dis J. 2012;31(3):297-301.

36. Musher DM. Editorial commentary: should 13-valent protein-conjugate pneumococcal vaccine be used routinely in adults? Clin Infect Dis. 2012;55(2):265-267.

37. Andrews NJ, Waight PA, George RC, Slack MP, Miller E. Impact and effectiveness of 23-valent pneumococcal polysaccharide vaccine against invasive pneumococcal disease in the elderly in England and Wales. Vaccine. 2012;30(48):6802-6808.

38. Prevention of pneumococcal disease: recommendations of the Advisory Committee on Immunization Practices (ACIP). MMWR Recomm Rep. 1997;46(RR-8):1-24.

39. Talbot TR, Hartert TV, Mitchel E, et al. Asthma as a risk factor for invasive pneumococcal disease. $N$ Engl J Med. 2005;352(20):2082-2090.

40. Nuorti JP, Butler JC, Farley MM, et al. Cigarette smoking and invasive pneumococcal disease. Active Bacterial Core Surveillance Team. N Engl J Med. 2000;342(10):681-689.

41. Centers for Disease Control and Prevention; Advisory Committee on Immunization Practices. Updated recommendations for prevention of invasive pneumococcal disease among adults using the 23 -valent pneumococcal polysaccharide vaccine (PPSV23). MMWR Morb Mortal Wkly Rep. 2010;59(34):1102-1106.

42. Badertscher N, Morell S, Rosemann T, Tandjung R. General practitioners' experiences, attitudes, and opinions regarding the pneumococcal vaccination for adults: a qualitative study. Int J Gen Med. 2012;5: 967-974.

43. Paradiso PR. Pneumococcal conjugate vaccine for adults: a new paradigm. Clin Infect Dis. 2012;55(2):259-264.

44. Richmond P, Borrow R, Miller E, et al. Meningococcal serogroup C conjugate vaccine is immunogenic in infancy and primes for memory. J Infect Dis. 1999;179(6):1569-1572.

45. Torling J, Hedlund J, Konradsen HB, Ortqvist A. Revaccination with the 23-valent pneumococcal polysaccharide vaccine in middleaged and elderly persons previously treated for pneumonia. Vaccine. 2003;22(1):96-103.

46. Black S, Shinefield H, Fireman B, et al. Efficacy, safety and immunogenicity of heptavalent pneumococcal conjugate vaccine in children. Northern California Kaiser Permanente Vaccine Study Center Group. Pediatr Infect Dis J. 2000;19(3):187-195.

47. Millar EV, Watt JP, Bronsdon MA, et al. Indirect effect of 7-valent pneumococcal conjugate vaccine on pneumococcal colonization among unvaccinated household members. Clin Infect Dis. 2008;47(8): 989-996.

48. Whitney CG, Farley MM, Hadler J, et al. Decline in invasive pneumococcal disease after the introduction of protein-polysaccharide conjugate vaccine. N Engl J Med. 2003;348(18):1737-1746.

49. French N, Gordon SB, Mwalukomo T, et al. A trial of a 7-valent pneumococcal conjugate vaccine in HIV-infected adults. N Engl J Med. 2010;362(9):812-822.
50. Dransfield MT, Nahm MH, Han MK, et al. Superior immune response to protein-conjugate versus free pneumococcal polysaccharide vaccine in chronic obstructive pulmonary disease. Am J Respir Crit Care Med. 2009;180(6):499-505.

51. Duggan ST. Pneumococcal polysaccharide conjugate vaccine (13-valent, adsorbed) (Prevenar 13 ${ }^{\circledR}$ ). Drugs. 2010;70(15):1973-1986.

52. Kieninger DM, Kueper K, Steul K, et al. Safety, tolerability, and immunologic noninferiority of a 13-valent pneumococcal conjugate vaccine compared to a 7 -valent pneumococcal conjugate vaccine given with routine pediatric vaccinations in Germany. Vaccine. 2010;28(25):4192-4203.

53. Plosker GL. 13-valent pneumococcal conjugate vaccine: a review of its use in infants, children, and adolescents. Paediatr Drugs. 2013;15(5):403-423.

54. Schwarz TF, Flamaing J, Rumke HC, et al. A randomized, double-blind trial to evaluate immunogenicity and safety of 13 -valent pneumococcal conjugate vaccine given concomitantly with trivalent influenza vaccine in adults aged $\geq 65$ years. Vaccine. 2011;29(32):5195-5202.

55. Pavia M, Bianco A, Nobile CG, Marinelli P, Angelillo IF. Efficacy of pneumococcal vaccination in children younger than 24 months: a meta-analysis. Pediatrics. 2009;123(6):e1103-e1110.

56. Moberley SA, Holden J, Tatham DP, Andrews RM. Vaccines for preventing pneumococcal infection in adults [review]. Cochrane Database Syst Rev. 2008;1:CD000422.

57. World Health Organization. 23-valent pneumococcal polysaccharide vaccine. WHO position paper. Wkly Epidemiol Rec. 2008;83(42): 373-384.

58. French N, Nakiyingi J, Carpenter LM, et al. 23-valent pneumococcal polysaccharide vaccine in HIV-1-infected Ugandan adults: double-blind, randomised and placebo controlled trial. Lancet. 2000;355(9221):2106-2111.

59. Metersky ML, Dransfield MT, Jackson LA. Determining the optimal pneumococcal vaccination strategy for adults: is there a role for the pneumococcal conjugate vaccine? Chest. 2010;138(3):486-490.

60. Romero-Steiner S, Musher DM, Cetron MS, et al. Reduction in functional antibody activity against Streptococcus pneumoniae in vaccinated elderly individuals highly correlates with decreased IgG antibody avidity. Clin Infect Dis. 1999;29(2):281-288.

61. Romero-Steiner S, Libutti D, Pais LB, et al. Standardization of an opsonophagocytic assay for the measurement of functional antibody activity against Streptococcus pneumoniae using differentiated HL-60 cells. Clin Diagn Lab Immunol. 1997;4(4):415-422.

62. Schuerman L, Prymula R, Henckaerts I, Poolman J. ELISA IgG concentrations and opsonophagocytic activity following pneumococcal protein D conjugate vaccination and relationship to efficacy against acute otitis media. Vaccine. 2007;25(11):1962-1968.

63. Jackson LA, Gurtman A, van Cleeff M, et al. Immunogenicity and safety of a 13-valent pneumococcal conjugate vaccine compared to a 23-valent pneumococcal polysaccharide vaccine in pneumococcal vaccine-naive adults. Vaccine. 2013;31(35):3577-3584

64. Jackson LA, Gurtman A, van Cleeff M, et al. Influence of initial vaccination with 13 -valent pneumococcal conjugate vaccine or 23 -valent pneumococcal polysaccharide vaccine on anti-pneumococcal responses following subsequent pneumococcal vaccination in adults 50 years and older. Vaccine. 2013;31(35):3594-3602.

65. Jackson LA, Gurtman A, Rice K, et al. Immunogenicity and safety of a 13-valent pneumococcal conjugate vaccine in adults 70 years of age and older previously vaccinated with 23 -valent pneumococcal polysaccharide vaccine. Vaccine. 2013;31(35):3585-3593.

66. Hak E, Grobbee DE, Sanders EA, et al. Rationale and design of CAPITA: a RCT of 13-valent conjugated pneumococcal vaccine efficacy among older adults. Neth J Med. 2008;66(9):378-383.

67. Pfizer. Pfizer completes pneumococcal pneumonia case accrual for CAPiTA, adult vaccine clinical trial of Prevenar 13* [press release]. New York, NY: Pfizer; 2013 [August 29]. Available from: http://press.pfizer. com/press-release/pfizer-completes-pneumococcal-pneumonia-caseaccrual-capita-adult-vaccine-clinical-tri. Accessed September 3, 2013. 
68. Pilishvili T, Lexau C, Farley MM, et al. Sustained reductions in invasive pneumococcal disease in the era of conjugate vaccine. J Infect Dis. 2010;201(1):32-41.

69. Clemens J, Shin S, Ali M. New approaches to the assessment of vaccine herd protection in clinical trials. Lancet Infect Dis. 2011;11(6): 482-487.

70. Paul Y. Herd immunity and herd protection. Vaccine. 2004;22(3-4): 301-302.

71. Fine P, Eames K, Heymann DL. "Herd immunity": a rough guide. Clin Infect Dis. 2011;52(7):911-916.

72. Kyaw MH, Rose CE Jr, Fry AM, et al. The influence of chronic illnesses on the incidence of invasive pneumococcal disease in adults. $J$ Infect Dis. 2005;192(3):377-386.

73. Cohen AL, Harrison LH, Farley MM, et al. Prevention of invasive pneumococcal disease among HIV-infected adults in the era of childhood pneumococcal immunization. AIDS. 2010;24(14):2253-2262.

74. Centers for Disease Control and Prevention (CDC). Use of 13-valent pneumococcal conjugate vaccine and 23 -valent pneumococcal polysaccharide vaccine for adults with immunocompromising conditions: recommendations of the Advisory Committee on Immunization Practices (ACIP). MMWR Morb Mortal Wkly Rep. 2012;61(40):816-819.

75. Hanna JN, Humphreys JL, Murphy DM, Smith HV. Invasive pneumococcal disease in non-indigenous people in north Queensland, 2001-2009. Med J Aust. 2010;193(7):392-396.

76. Miller E, Andrews NJ, Waight PA, Slack MP, George RC. Herd immunity and serotype replacement 4 years after seven-valent pneumococcal conjugate vaccination in England and Wales: an observational cohort study. Lancet Infect Dis. 2011;11(10):760-768.

77. Ardanuy C, Tubau F, Pallares R, et al. Epidemiology of invasive pneumococcal disease among adult patients in Barcelona before and after pediatric 7-valent pneumococcal conjugate vaccine introduction, 1997-2007. Clin Infect Dis. 2009;48(1):57-64.

78. Fenoll A, Granizo JJ, Aguilar L, et al. Temporal trends of invasive Streptococcus pneumoniae serotypes and antimicrobial resistance patterns in Spain from 1979 to 2007. J Clin Microbiol. 2009;47(4): 1012-1020.

79. Lepoutre A, Varon E, Georges S, Gutmann L, Levy-Bruhl D. Impact of infant pneumococcal vaccination on invasive pneumococcal diseases in France, 2001-2006. Euro Surveill. 2008;13(35):pii18962.

80. Hanquet G, Kissling E, Fenoll A, et al. Pneumococcal serotypes in children in four European countries. Emerg Infect Dis. 2010;16(9): 1428-1439.

81. Rozenbaum MH, Hoek AJ, Hak E, Postma MJ. Huge impact of assumptions on indirect effects on the cost-effectiveness of routine infant vaccination with 7-valent conjugate vaccine (Prevnar). Vaccine. 2010;28(12):2367-2369.

82. Tyo KR, Rosen MM, Zeng W, et al. Cost-effectiveness of conjugate pneumococcal vaccination in Singapore: comparing estimates for 7-valent, 10-valent, and 13-valent vaccines. Vaccine. 2011;29(38): 6686-6694.
83. ACIP Adult Immunization Work Group, Bridges CB, Woods L, CoyneBeasley $\mathrm{T}$ and Centers for Disease Control and Prevention (CDC). Advisory Committee on Immunization Practices (ACIP) recommended immunization schedule for adults aged 19 years and older - United States, 2013. MMWR Surveill Summ. 2013;62(Suppl 1):9-19.

84. Committee for Medicinal Products for Human Use (CHMP). Summary of Opinion: Prevenar 13. London: European Medicines Agency; 2013. Available from: http://www.ema.europa.eu/docs/en_GB/document library/Summary_of_opinion/human/001104/WC500143813.pdf. Accessed September 3, 2013.

85. Sun HK, Nicolau DP, Kuti JL. Resource utilization of adults admitted to a large urban hospital with community-acquired pneumonia caused by Streptococcus pneumoniae. Chest. 2006;130(3):807-814.

86. Lee KK, Rinaldi F, Chan MK, et al. Economic evaluation of universal infant vaccination with $7 \mathrm{vPCV}$ in Hong Kong. Value Health. 2009;12(Suppl 3):S42-S48.

87. Sohn HS, Suh DC, Jang E, Kwon JW. Economic evaluation of childhood 7-valent pneumococcal conjugate vaccination in Korea. J Manag Care Pharm. 2010;16(1):32-45.

88. Rubin JL, McGarry LJ, Strutton DR, et al. Public health and economic impact of the 13-valent pneumococcal conjugate vaccine (PCV13) in the United States. Vaccine. 2010;28(48):7634-7643.

89. Smith KJ, Wateska AR, Nowalk MP, Raymund M, Nuorti JP, Zimmerman RK. Cost-effectiveness of adult vaccination strategies using pneumococcal conjugate vaccine compared with pneumococcal polysaccharide vaccine. JAMA. 2012;307(8):804-812.

90. Smith KJ, Nowalk MP, Raymund M, Zimmerman RK. Cost-effectiveness of pneumococcal conjugate vaccination in immunocompromised adults. Vaccine. 2013;31(37):3950-3956.

91. Lian LP. Bridging the gap: the PDV programme at Tan Tock Seng Hospital [abstract]. Ann Acad Med Singapore. 2011;40(Suppl 7):S4.

92. Centers for Disease Control and Prevention. Adult vaccination coverage - United States, 2010. MMWR Morb Mortal Wkly Rep. 2012;61(4):66-72.

93. Adult immunization: shots to save lives [webpage on the internet]. Washington, DC: Trust for America's Health; 2010. Available from: http:// healthyamericans.org/report/73/adultimmunization-2010. Accessed September 3, 2013.

94. Centers for Disease Control and Prevention. Pneumococcal disease. In: Atkinson W, Wolfe S, Hamborsky J, editors. Epidemiology and Prevention of Vaccine-Preventable Diseases. 12th ed. Washington, DC: Public Health Foundation; 2012:233-248.

95. Nowalk MP, Nolan BA, Nutini J, et al. Success of the 4 Pillars Toolkit for influenza and pneumococcal vaccination in adults. J Healthc Qual. Epub June 18, 2013.

96. Middleton DB, Lin CJ, Smith KJ, et al. Economic evaluation of standing order programs for pneumococcal vaccination of hospitalized elderly patients. Infect Control Hosp Epidemiol. 2008;29(5):385-394.
International Journal of General Medicine

\section{Publish your work in this journal}

The International Journal of General Medicine is an international, peer-reviewed open-access journal that focuses on general and internal medicine, pathogenesis, epidemiology, diagnosis, monitoring and treatment protocols. The journal is characterized by the rapid reporting of reviews, original research and clinical studies across all disease areas.

\section{Dovepress}

A key focus is the elucidation of disease processes and management protocols resulting in improved outcomes for the patient. The manuscript management system is completely online and includes a very quick and fair peer-review system. Visit http://www.dovepress.com/ testimonials.php to read real quotes from published authors. 